\title{
Performance Analysis of MAC for Power-Line Communications
}

\author{
Christina Vlachou \\ EPFL, Switzerland \\ christina.vlachou@epfl.ch \\ Julien Herzen \\ EPFL, Switzerland \\ julien.herzen@epfl.ch
}

\author{
Albert Banchs \\ Universidad Carlos III de \\ Madrid, Spain \\ banchs@it.uc3m.es \\ Patrick Thiran \\ EPFL, Switzerland \\ patrick.thiran@epfl.ch
}

\begin{abstract}
We investigate the IEEE 1901 MAC protocol, the dominant protocol for high data rate power-line communications. 1901 employs a CSMA/CA mechanism similar to - but much more complex than - the backoff mechanism of 802.11. Because of this extra complexity, and although this mechanism is the only widely used MAC layer for power-line networks, there are few analytical results on its performance. We propose a model for the 1901 MAC that comes in the form of a single fixed-point equation for the collision probability. We prove that this equation admits a unique solution, and we evaluate the accuracy of our model by using simulations.
\end{abstract}

\section{Keywords}

Power-line communications; CSMA/CA; analysis.

\section{Categories and Subject Descriptors}

C.4 [Performance of Systems]: Modeling techniques

\section{BACKGROUND}

Due to the shared nature of power lines, the vast majority of devices employ a multiple-access scheme based on CSMA/CA, which is specified by the IEEE 1901 standard [1]. We first present the relevant aspects of this protocol. The CSMA/CA process of 1901 uses two counters: the backoff counter $(B C)$ and the deferral counter $(D C)$. In addition, there are four backoff stages. Upon the arrival of a new packet, a transmitting station starts at backoff stage 0 . It then draws the backoff counter $B C$ uniformly at random in $\left\{0, \ldots, C W_{0}-1\right\}$, where $C W_{0}$ refers to the contention window used at backoff stage 0 . Similarly to $802.11, B C$ is decreased by 1 at each time slot if the station senses the medium to be idle (i.e., below the carrier-sensing threshold), and $B C$ is frozen when the medium is sensed busy, in

Permission to make digital or hard copies of part or all of this work for personal or classroom use is granted without fee provided that copies are not made or distributed for profit or commercial advantage, and that copies bear this notice and the full citation on the first page. Copyrights for third-party components of this work must be honored. For all other uses, contact the owner/author(s). Copyright is held by the author/owner(s).

SIGMETRICS'14, June 16-20, 2014, Austin, Texas, USA.

ACM 978-1-4503-2789-3/14/06.

http://dx.doi.org/10.1145/2591971.2592033. which case $B C$ will be also decreased by 1 once the medium is sensed idle again. When $B C$ reaches 0 , the station attempts to transmit the packet. Also similarly to 802.11 , the station jumps to the next backoff stage if the transmission fails (unless it is already at the last backoff stage, in which case it re-enters this backoff stage). When entering backoff stage $i$, a station draws $B C$ uniformly at random in $\left\{0, \ldots, C W_{i}-1\right\}$, where $C W_{i}$ is the contention window at backoff stage $i$, and the process is repeated. The default $C W_{i}$ values are $C W_{i} \in\{8,16,32,64\}$ for 1901 .

Now, the main difference between 1901 and 802.11 is that a 1901 station might enter the next backoff stage even if it did not attempt a transmission. The mechanism for deciding when this occurs uses the deferral counter $D C$, and works as follows. When entering backoff stage $i, D C$ is set at an an initial $D C$ value $d_{i}$ (the defaults being $\{0,1,3,15\}$ [1]). After having sensed the medium busy, a station decreases $D C$ by 1 (in addition to $B C$ ). If the medium is sensed busy and $D C=0$, then the station jumps to the next backoff stage (or re-enters the last backoff stage, if it is already at this stage), and it re-draws $B C$ without attempting a transmission.

The deferral counter significantly increases the complexity of the 1901 backoff procedure. To the best of our knowledge, the only work that analyzes this procedure is [2], and uses a Markov chain. To compute the collision probability $\gamma$, a costly system of more than a thousand non-linear equations needs to be solved [2]. Moreover, the authors do not study whether this system of equations has a unique solution. We introduce here a simplification of [2] and prove that our model admits a unique solution. Our model can be later employed to investigate enhancements of the 1901 MAC.

\section{ANALYSIS}

We investigate the 1901 CSMA/CA protocol under the following assumptions. First, there are $N$ stations in a single contention domain. Second, there is no packet loss or error due to the physical layer. Third, the stations have an infinite retry limit.

Our modeling assumptions are similar to the ones in [3] and are referred to as the decoupling assumption. We assume that the backoff process of a station is independent of the aggregate attempt process of the other $N-1$ stations and that transmission attempts experience a fixed collision probability $\gamma$. In addition, $\gamma$ refers to the probability that the medium is busy at any time slot. Finally, the stations 
attempt a transmission in each time slot, with a constant transmission probability equal to the average attempt rate $\tau$.

A 1901 station with $D C$ initially equal to $d_{i}$ can change its backoff stage either (i) after attempting a transmission or (ii) due to sensing the medium busy $d_{i}+1$ times. We need to compute the probabilities of the events (i) and (ii). To this end, we introduce $x_{k}$, the probability that a station at backoff stage $i$ jumps to the next backoff stage $i+1$ in $k$ or fewer time slots due to (ii). The event that (ii) happens during the first $k$ slots follows the binomial distribution $\operatorname{Bin}(k, \gamma)$, whence $x_{k}=\sum_{j=d_{i}+1}^{k}\left(\begin{array}{c}k \\ j\end{array}\right) \gamma^{j}(1-\gamma)^{k-j}$.

Let us write $b c_{i}$ for the expected number of time slots spent by a station at backoff stage $i$. Now, recall that when entering stage $i$, the stations draw a backoff counter $B C$ uniformly at random in $\left\{0, \ldots, C W_{i}-1\right\}$. Let $k$ denote this initial value of $B C$, and $d_{i}$ be the value of $D C$ when the station enters stage $i$. If $k>d_{i}$, then event (i) occurs with probability $\left(1-x_{k}\right)$, in which case the station spends $(k+1)$ slots in stage $i$ (the $(k+1)$ th slot being used for transmission), whence event (ii) occurs at slot $j$, with $d_{i}+1 \leq j \leq k$, with probability $\left(x_{j}-x_{j-1}\right)$, in which case the station spends $j$ slots in stage $i$. Finally, if $k \leq d_{i}$, then (ii) cannot happen and (i) always takes place. It follows that $b c_{i}$ is given by

$$
\begin{aligned}
b c_{i} & =\frac{1}{C W_{i}} \sum_{k=d_{i}+1}^{C W_{i}-1}\left[(k+1)\left(1-x_{k}\right)+\sum_{j=d_{i}+1}^{k} j\left(x_{j}-x_{j-1}\right)\right] \\
& +\frac{\left(d_{i}+1\right)\left(d_{i}+2\right)}{2 C W_{i}} .
\end{aligned}
$$

Let $R$ be a random variable describing the number of transmission attempts experienced by a successfully transmitted packet and let $X$ be a random variable describing the total number of slots spent in backoff for a successfully transmitted packet. Then by using the renewal-reward theorem as in [3], the average attempt rate can be computed as

$$
\tau=\mathbb{E}[R] / \mathbb{E}[X]
$$

We now compute separately $\mathbb{E}[R]$ and $\mathbb{E}[X]$. Let $t_{i}$ be the probability that a station at backoff stage $i$ ends this backoff stage by attempting a transmission. Using a similar reasoning as for (1), $t_{i}$ is given by

$$
t_{i}=\frac{d_{i}+1}{C W_{i}}+\sum_{k=d_{i}+1}^{C W_{i}-1} \frac{1}{C W_{i}}\left(1-x_{k}\right) .
$$

Let $s_{i}=(1-\gamma) t_{i}$ be the probability that a station at backoff stage $i$ ends this stage due to a successful transmission and let $m$ be the total number of backoff stages. We express $\mathbb{E}[R]$ and $\mathbb{E}[X]$ as a function of all $b c_{i}, t_{i}, s_{i}$. The expected number of backoff slots per successfully transmitted packet is

$$
\mathbb{E}[X]=\sum_{i=0}^{m-2} b c_{i} \prod_{j=0}^{i-1}\left(1-s_{j}\right)+\prod_{i=0}^{m-2}\left(1-s_{i}\right) \frac{b c_{m-1}}{s_{m-1}} .
$$

Finally, the expected number of transmission attempts per successfully transmitted packet is

$$
\mathbb{E}[R]=\sum_{i=0}^{m-2} t_{i} \prod_{j=0}^{i-1}\left(1-s_{j}\right)+\prod_{i=0}^{m-2}\left(1-s_{i}\right) \frac{t_{m-1}}{s_{m-1}}=\frac{1}{1-\gamma} .
$$

Let $G(\gamma)=\mathbb{E}[R] / \mathbb{E}[X]$. Now, $\gamma$ is the probability that at least one other station transmits and it can be expressed as a function of $\tau: \gamma=\Gamma(\tau)=1-(1-\tau)^{N-1}$. The composition of the two functions above yields the fixed-point equation for the collision probability: $\gamma=\Gamma(G(\gamma))$. Theorem 1 below establishes the uniqueness of the solution of $\gamma=\Gamma(G(\gamma))$.

THEOREM 1. The fixed-point equation $\gamma=\Gamma(G(\gamma))$ has a unique solution if $C W_{i+1}>2 C W_{i}-d_{i}-1,0 \leq i \leq m-1$.

\section{PERFORMANCE EVALUATION}

We use simulations to evaluate the 1901 performance. We wrote a custom simulator in Matlab, which implements the full CSMA/CA mechanism of 1901.

Figure 1 presents the collision probability and the normalized throughput of the network obtained by simulations and our model. We observe that the accuracy of the model improves as $N$ increases. We attribute the reduced accuracy when $N$ is small to the decoupling assumption: For small $N$, the stations are coupled in 1901 because they change their state when some other station transmits. We leave the investigation of the validity of the decoupling assumption for future work.
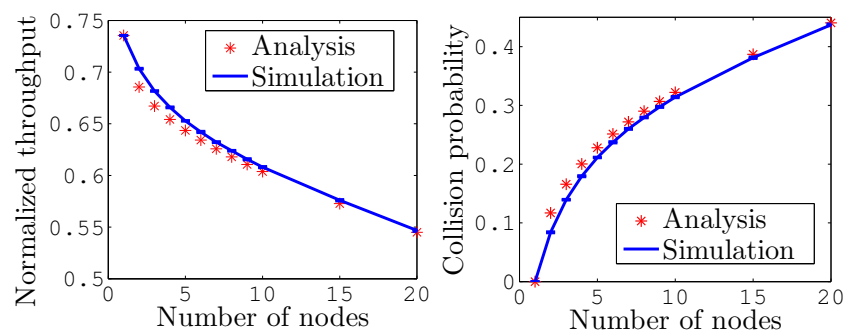

Figure 1: Performance metrics obtained by simulation and our model for the 1901 CSMA/CA method.

\section{CONCLUSION}

We have proposed a model for analyzing the performance of IEEE 1901 CSMA/CA method, which is the dominant protocol for power-line networks. Our model is equivalent to the Markov chain in [2], but its simplicity allows us to prove the uniqueness of the solution for the collision probability.

Our future research directions include enhancements of 1901 based on the model presented in this paper. Furthermore, we plan to investigate the validity of the decoupling assumption for 1901, as we observed that the accuracy of the model suffers when there are few stations in the network.

\section{Acknowledgments}

This work is financially supported by a grant of the SmartWorld project of the Hasler Foundation, Bern, Switzerland.

\section{REFERENCES}

[1] IEEE Standard for Broadband over Power Line Networks: Medium Access Control and Physical Layer Specifications. IEEE Std 1901-2010, pages 1-1586, 2010.

[2] M. Chung, M. Jung, T. Lee, and Y. Lee. Performance analysis of HomePlug 1.0 MAC with CSMA/CA. Selected Areas in Communications, IEEE Journal on, 24(7):1411-1420, 2006.

[3] A. Kumar, E. Altman, D. Miorandi, and M. Goyal. New Insights From a Fixed-Point Analysis of Single Cell IEEE 802.11 WLANs. Networking, IEEE/ACM Transactions on, 15(3):588-601, june 2007. 